\title{
Weighted Hermite-Hadamard type inclusions for products of co-ordinated convex interval-valued functions
}

\author{
Hasan Kara', Hüseyin Budak', Muhammad Aamir Ali², Mehmet Zeki Sarikaya' and Yu-Ming Chu ${ }^{3 *}$ (D)
}

\section{"Correspondence:}

chuyuming@zjhu.edu.cn

${ }^{3}$ Department of Mathematics,

Huzhou University, Huzhou 313000

China

Full list of author information is

available at the end of the article

\section{Springer}

\begin{abstract}
In this paper, we establish some Hermite-Hadamard-Fejér type inclusions for the product of two co-ordinated convex interval-valued functions. These inclusions are generalizations of some results given in earlier works.
\end{abstract}

MSC: 26D07; 26D10; 26D15; 26B15; 26B25

Keywords: Hermite-Hadamard inclusions; Convex interval-valued functions; Co-ordinated convex interval-valued functions

\section{Introduction}

Over the last century, integral inequalities have attracted the interest of a good many researchers because of the importance in applied and pure mathematics. For example, Hermite-Hadamard inequalities, based on convex functions, have an important place in many areas of mathematics, specifically optimization theory. These inequalities, introduced by C. Hermite and J. Hadamard, express that if $\varpi: I \rightarrow \mathbb{R}$ is a convex mapping on the interval $I$ of real numbers and $c, d \in I$ with $c<d$, then

$$
\varpi\left(\frac{c+d}{2}\right) \leq \frac{1}{d-c} \int_{c}^{d} \varpi(x) d x \leq \frac{\varpi(c)+\varpi(d)}{2} .
$$

If $\varpi$ is concave, both inequalities hold in the opposite direction. The best known results associated with these inequalities are midpoint and trapezoid inequalities which are frequently used in special means and estimation errors (see [16, 25]). After that, the authors also gave the fractional version of inequality (1.1) in [49]. For instance, the weighted version of inequality (1.1), which is also named Hermite-Hadamard-Fejér inequality, was established by Fejér in [18] as follows.

Theorem 1 Suppose that $\varpi:[c, d] \rightarrow \mathbb{R}$ is a convex function, and let $\phi:[c, d] \rightarrow \mathbb{R}$ be nonnegative, integrable, and symmetric about $x=\frac{c+d}{2}(i . e . \phi(x)=\phi(c+d-x))$. Then we

(c) The Author(s) 2021. This article is licensed under a Creative Commons Attribution 4.0 International License, which permits use, sharing, adaptation, distribution and reproduction in any medium or format, as long as you give appropriate credit to the original author(s) and the source, provide a link to the Creative Commons licence, and indicate if changes were made. The images or other third party material in this article are included in the article's Creative Commons licence, unless indicated otherwise in a credit line to the material. If material is not included in the article's Creative Commons licence and your intended use is not permitted by statutory regulation or exceeds the permitted use, you will need to obtain permission directly from the copyright holder. To view a copy of this licence, visit http://creativecommons.org/licenses/by/4.0/. 
have the inequality

$$
\varpi\left(\frac{c+d}{2}\right) \int_{c}^{d} \phi(x) d x \leq \int_{c}^{d} \varpi(x) \phi(x) d x \leq \frac{\varpi(c)+\varpi(d)}{2} \int_{c}^{d} \phi(x) d x .
$$

Many mathematicians derived some generalizations and new results involving fractional integrals regarding inequality (1.2) to obtain new bounds for the left- and right-hand sides of inequality (1.2) (see $[17,47,48]$ ). In addition to all these generalizations, a good many of authors have worked on Hermite-Hadamard type inequalities for the product of two convex functions in recent years. Moreover, some of them obtained Hermite-Hadamard type results and included fractional integrals in their works. For instance, Pachpatte provided novel inequalities for the product of two nonnegative and convex mappings in [40]. After that, some authors examined how the results were obtained by multiplying two mappings selected from various convex function classes in the references $[3,10-12,21,22,26,50-$ 52]. What is more, some inequalities involving the product of two co-ordinated convex mappings were observed by Latif and Alomari in [27]. Thereafter, Ozdemir et al. [38, 39] deduced more general versions of the inequalities presented in [27] by considering the product of two co-ordinated $s$-convex and the product of two co-ordinated $h$-convex mappings. In [6], by using the products of two co-ordinated convex mappings, new HermiteHadamard type results including fractional integrals were proved by Budak and Sarıkaya.

On the other hand, interval analysis is handled as one of the methods for solving interval uncertainty; it is an important material which is used in mathematical and computer models. Although this theory has a long history which may be dated back to Archimedes' calculation of the circumference of a circle, a considerable study had not been published in this field until 1950s. The first book [33] about interval analysis was published by Ramon E. Moore, known as the pioneer of interval calculus, in 1966. Thereafter, a great many researchers started to investigate the theories and applications of interval analysis. Recently, many authors have focused on integral inclusions obtained by using interval-valued functions. For example, Sadowska [46] established the Hermite-Hadamard inclusion for setvalued functions, that is, a more general version of interval-valued mappings, as follows.

Theorem $2([46])$ Suppose that $\digamma:[\varrho, \varsigma] \rightarrow \mathbb{R}_{\mathcal{I}}^{+}$is an interval-valued convex function such that $\digamma(\vartheta)=[\underline{\digamma}(\vartheta), \bar{\digamma}(\vartheta)]$. Then we have the inclusions

$$
\digamma\left(\frac{\varrho+\varsigma}{2}\right) \supseteq \frac{1}{\varsigma-\varrho}(I R) \int_{\varrho}^{\varsigma} \digamma(x) d x \supseteq \frac{\digamma(\varrho)+\digamma(\varsigma)}{2} .
$$

Furthermore, well-known inclusions such as Ostrowski, Minkowski, and Beckenbach and some of their applications have been provided by considering interval-valued functions in $[8,9,19,44]$. In addition, some inclusions involving interval-valued RiemannLiouville fractional integrals have been derived by Budak et al. in [7]. In [28], Liu et al. gave the definition of interval-valued harmonically convex functions, and so they have some Hermite-Hadamard type inclusions including interval fractional integrals. For more details about this topic, you can look over the references [13, 14, 32, 34-36, 45, 55, 56].

The general structure of this paper consists of four main sections including introduction. In this section, we give some necessary inclusions and the concept of interval analysis, and we also mention some related works in the literature. In Sect. 2, some basic information about the interval calculus which forms the basis of this work is presented. In Sect. 3, 
we provide Fejér type inclusions for the product of interval-valued convex functions, and we examine the relation between our results and the inclusions presented in the earlier works. Finally, we establish interval-valued Fejér type inclusions including fractional integrals by applying the inclusions given in Sect. 3 to interval-valued fractional integrals in Sect. 4. Briefly, the most important property of this study is that it contains interval-valued Fejér type inclusions for classical and fractional integrals. We note that the opinion and technique of this work may inspire new research in this area.

\section{Preliminaries of interval calculus and some inclusions}

In this part, we give some necessary notions and notations related to interval analysis, which forms the basis of this paper. The set of all closed intervals of $\mathbb{R}$, the set of all closed positive intervals of $\mathbb{R}$, and the set of all closed negative intervals of $\mathbb{R}$ are denoted by $\mathbb{R}_{\mathcal{I}}, \mathbb{R}_{\mathcal{I}}^{+}$, and $\mathbb{R}_{\mathcal{I}}^{-}$, respectively. We also suppose that $\Delta=[\varrho, \zeta] \times[\zeta, \iota]$. For more details regarding the interval analysis, interested readers can see $[1,15,30,41]$.

Definition 1 ([46]) Let $\digamma:[\varrho, \varsigma] \rightarrow \mathbb{R}_{\mathcal{I}}^{+}$be an interval-valued function such that $\digamma(\vartheta)=$ $[\underline{\digamma}(\vartheta), \bar{\digamma}(\vartheta)]$. We say that $\digamma$ is a convex interval-valued function if, for all $\xi, \eta \in[\varrho, \varsigma]$ and $\vartheta \in(0,1)$, we have

$$
\vartheta \digamma(\xi)+(1-\vartheta) \digamma(\eta) \subseteq \digamma(\vartheta \xi+(1-\vartheta) \eta) .
$$

Definition 2 ([54]) Suppose that $h:[\zeta, \iota] \rightarrow \mathbb{R}$ is a nonnegative function with $(0,1) \subseteq$ $[\zeta, \iota], h \neq 0$ and $\digamma:[\varrho, \zeta] \rightarrow \mathbb{R}_{\mathcal{I}}^{+}$is an interval-valued function such that $\digamma(\vartheta)=[\underline{\digamma}(\vartheta)$, $\bar{\digamma}(\vartheta)]$. We say that $\digamma$ is an $h$-convex interval-valued function if, for all $\xi, \eta \in[\varrho, \varsigma]$ and $\vartheta \in(0,1)$, we have

$$
h(\vartheta) \digamma(\xi)+h(1-\vartheta) \digamma(\eta) \subseteq \digamma(\vartheta \xi+(1-\vartheta) \eta) .
$$

Remark 1 If we set $h(\vartheta)=\vartheta$ in Definition 2, then Definition 2 reduces to Definition 1 . Similarly, if we choose $h(\vartheta)=\vartheta^{s}$ in Definition 2, then Definition 2 transforms into $s$-convex interval-valued functions given in [2].

In [42], Piatek gave the notion of the integral of interval-valued functions and provided its relation with the Riemann integral in the following form.

Theorem 3 Assume that $\digamma:[\varrho, \varsigma] \rightarrow \mathbb{R}_{\mathcal{I}}^{+}$is an interval-valued function such that $\digamma(\vartheta)=$ $[\underline{\digamma}(\vartheta), \bar{\digamma}(\vartheta)]$. A function $\digamma \in \mathcal{I R}_{([\varrho, 5])}$ if and only if $\underline{\digamma}(\vartheta), \bar{\digamma}(\vartheta) \in \mathcal{R}_{([\varrho, 5])}$ and

$$
(I R) \int_{\varrho}^{\zeta} \digamma(\vartheta) d \vartheta=\left[\int_{\varrho}^{\zeta} \underline{\digamma}(\vartheta) d \vartheta, \int_{\varrho}^{\zeta} \bar{\digamma}(\vartheta) d \vartheta\right]
$$

where $\mathcal{I} \mathcal{R}_{([\varrho, 5])}$ and $\mathcal{R}_{([\varrho, 5])}$ denote all Riemann integrable interval-valued functions and Riemann integrable functions, respectively.

In [54], Zhao et al. gave the following inclusions of Hermite-Hadamard type for $h$ convex interval-valued functions by using Theorem 3 . 
Theorem 4 Let $\digamma:[\varrho, \varsigma] \rightarrow \mathbb{R}_{\mathcal{I}}^{+}$be an interval-valued function such that $\digamma(\vartheta)=$ $[\underline{\digamma}(\vartheta), \bar{\digamma}(\vartheta)]$ and $\digamma \in \mathcal{I R}_{([\varrho, \zeta])}, h:[0,1] \rightarrow \mathbb{R}$ be a nonnegative function and $h\left(\frac{1}{2}\right) \neq 0$. If $\digamma$ is an h-convex interval-valued function, then the following inclusion holds:

$$
\frac{1}{2 h\left(\frac{1}{2}\right)} \digamma\left(\frac{\varrho+\varsigma}{2}\right) \supseteq \frac{1}{\varsigma-\varrho}(I R) \int_{\varrho}^{\varsigma} \digamma(\xi) d \xi \supseteq[\digamma(\varrho)+\digamma(\varsigma)] \int_{0}^{1} h(\vartheta) d \vartheta
$$

\section{Remark 2}

(i) If $h(\vartheta)=\vartheta$, then inclusion (2.2) reduces to (1.3).

(ii) If $h(\vartheta)=\vartheta^{s}$, then (2.2) reduces to the following result:

$$
2^{s-1} \digamma\left(\frac{\varrho+\varsigma}{2}\right) \supseteq \frac{1}{\varsigma-\varrho}(I R) \int_{\varrho}^{\varsigma} \digamma(\xi) d \xi \supseteq \frac{\digamma(\varrho)+\digamma(\varsigma)}{s+1},
$$

which is obtained by Osuna-Gómez et al. in [37].

Now, let us give the notations $\mathcal{A}_{k}(\xi ; m, n)$ and $\mathcal{B}_{k}(\xi ; m, n)$ used throughout the study:

$$
\begin{aligned}
& \mathcal{A}_{k}(\xi ; m, n)=\int_{m}^{n}(n-\xi)^{2} w_{k}(\xi) d \xi \quad \text { and } \\
& \mathcal{B}_{k}(\xi ; m, n)=\int_{m}^{n}(n-\xi)(\xi-m) w_{k}(\xi) d \xi, \quad k=1,2,
\end{aligned}
$$

where $w_{k}:[\varrho, \varsigma] \rightarrow \mathbb{R}$ is a function.

Budak et al. presented the following results.

Theorem 5 ([5]) Suppose that $w_{1}:[\varrho, \varsigma] \rightarrow \mathbb{R}$ is nonnegative, integrable, and symmetric about $\xi=\frac{\varrho+\varsigma}{2}\left(\right.$ i.e. $\left.w_{1}(\xi)=w_{1}(\varrho+\varsigma-\xi)\right)$. If $\digamma, \Omega:[\varrho, \varsigma] \rightarrow \mathbb{R}_{\mathcal{I}}^{+}$are two intervalvalued, nonnegative, and convex interval-valued functions such that $\digamma(\vartheta)=[\underline{\digamma}(\vartheta), \bar{\digamma}(\vartheta)]$ and $\Omega(\vartheta)=[\underline{\Omega}(\vartheta), \bar{\Omega}(\vartheta)]$, then we have the following inclusion:

$$
(I R) \int_{\varrho}^{\varsigma} \digamma(\xi) \Omega(\xi) w_{1}(\xi) d \xi \supseteq \frac{\mathbf{M}(\varrho, \varsigma)}{(\varsigma-\varrho)^{2}} \mathcal{A}_{1}(\xi ; \varrho, \varsigma)+\frac{\mathbf{N}(\varrho, \varsigma)}{(\varsigma-\varrho)^{2}} \mathcal{B}_{1}(\xi ; \varrho, \varsigma),
$$

where

$$
\mathbf{M}(\varrho, \varsigma)=\digamma(\varrho) \Omega(\varrho)+\digamma(\varsigma) \Omega(\varsigma) \text { and } \mathbf{N}(\varrho, \varsigma)=\digamma(\varrho) \Omega(\varsigma)+\digamma(\varsigma) \Omega(\varrho) \text {. }
$$

Theorem 6 ([5]) Suppose that the conditions of Theorem 5 hold, then we have the following inclusion:

$$
\begin{aligned}
& 2 \digamma\left(\frac{\varrho+\varsigma}{2}\right) \Omega\left(\frac{\varrho+\varsigma}{2}\right) \int_{\varrho}^{\varsigma} w_{1}(\xi) d \xi \\
& \quad \supseteq(I R) \int_{\varrho}^{\varsigma} \digamma(\xi) \Omega(\xi) w_{1}(\xi) d \xi+\frac{\mathbf{M}(\varrho, \varsigma)}{(\varsigma-\varrho)^{2}} \mathcal{B}_{1}(\xi ; \varrho, \varsigma)+\frac{\mathbf{N}(\varrho, \varsigma)}{(\varsigma-\varrho)^{2}} \mathcal{A}_{1}(\xi ; \varrho, \varsigma),
\end{aligned}
$$

where $\mathbf{M}(\varrho, \varsigma)$ and $\mathbf{N}(\varrho, \varsigma)$ are defined as in Theorem 5. 
Motivated by the continuing studies, the authors introduced the fractional integrals for interval-valued functions and proved some associated inclusions of Hermite-Hadamard type.

Definition $3([7,29])$ Assume that $\digamma:[\varrho, \varsigma] \rightarrow \mathbb{R}_{\mathcal{I}}^{+}$is an interval-valued function such that $\digamma(\vartheta)=[\underline{\digamma}(\vartheta), \bar{\digamma}(\vartheta)]$. Then interval-valued Riemann-Liouville integrals $\mathcal{J}_{\varrho+}^{\alpha} \digamma$ and $\mathcal{J}_{\zeta-}^{\alpha} \digamma$ of order $\alpha>0$ with $\varrho \geq 0$ are defined by

$$
\mathcal{J}_{\varrho+}^{\alpha} \digamma(\xi)=\frac{1}{\Gamma(\alpha)}(I R) \int_{\varrho}^{\xi}(\xi-\vartheta)^{\alpha-1} \digamma(\vartheta) d \vartheta, \quad \xi>\varrho,
$$

and

$$
\mathcal{J}_{\zeta-}^{\alpha} \digamma(\xi)=\frac{1}{\Gamma(\alpha)}(I R) \int_{\xi}^{\varsigma}(\vartheta-\xi)^{\alpha-1} \digamma(\vartheta) d \vartheta, \quad \xi<\varsigma,
$$

respectively. Here, $\Gamma(\alpha)$ is the gamma function and $\mathcal{J}_{\varrho^{+}}^{0} \digamma(\xi)=\mathcal{J}_{\zeta_{-}}^{0} \digamma(\xi)=\digamma(\xi)$.

Theorem $7([7])$ If $\digamma=[\underline{\digamma}(\vartheta), \bar{\digamma}(\vartheta)]:[\varrho, \varsigma] \rightarrow \mathbb{R}_{\mathcal{I}}$ is an interval-valued function, then one has

$$
\mathcal{J}_{\varrho^{+}}^{\alpha} \digamma(\xi)=\left[I_{\varrho^{+}}^{\alpha} \underline{\digamma}(\xi), I_{\varrho^{+}}^{\alpha} \bar{\digamma}(\xi)\right]
$$

and

$$
\mathcal{J}_{\varsigma_{-}}^{\alpha} \digamma(\xi)=\left[I_{\varsigma^{-}}^{\alpha} \underline{\digamma}(\xi), I_{\varsigma_{-}}^{\alpha} \bar{\digamma}(\xi)\right]
$$

where $I_{\varrho^{+}}^{\alpha}$ and $I_{5_{-}}^{\alpha}$ are left-sided and right-sided Riemann-Liouville fractional integrals of the functions $\digamma:[\varrho, \varsigma] \rightarrow \mathbb{R}$, respectively $($ see $[20,24,31,43])$.

Theorem $8([7])$ Let $\digamma=[\underline{\digamma}(\vartheta), \bar{\digamma}(\vartheta)]:[\varrho, \varsigma] \rightarrow \mathbb{R}_{\mathcal{I}}^{+}$be a convex interval-valued function and $\alpha>0$, then we have the following inclusion:

$$
\digamma\left(\frac{\varrho+\varsigma}{2}\right) \supseteq \frac{\Gamma(1+\alpha)}{2(\varsigma-\varrho)^{\alpha}}\left[\mathcal{J}_{\varrho+}^{\alpha} \digamma(\varsigma)+\mathcal{J}_{\varsigma-}^{\alpha} \digamma(\varrho)\right] \supseteq \frac{\digamma(\varrho)+\digamma(\varsigma)}{2} .
$$

In addition to all these results, Liu et al. refined Hermite-Hadamard type inclusions for interval-valued mappings in [28].

Theorem 9 ([28]) Suppose that $w:[\varrho, \varsigma] \rightarrow \mathbb{R}$ is nonnegative, integrable, and symmetric about $\xi=\frac{\varrho+\varsigma}{2}($ i.e. $w(\xi)=w(\varrho+\varsigma-\xi))$. If $\digamma=[\underline{\digamma}(\vartheta), \bar{\digamma}(\vartheta)]:[\varrho, \varsigma] \rightarrow \mathbb{R}_{\mathcal{I}}^{+}$is a convex interval-valued function, then we have the following inclusion:

$$
\begin{aligned}
& \digamma\left(\frac{\varrho+\varsigma}{2}\right)\left[I_{\varrho+}^{\alpha} w(\varsigma)+I_{\varsigma_{-}}^{\alpha} w(\varrho)\right] \\
& \supseteq \supseteq\left[\mathcal{J}_{\varrho+}^{\alpha} \digamma(\varsigma) w(\varsigma)+\mathcal{J}_{\varsigma-}^{\alpha} \digamma(\varrho) w(\varrho)\right] \\
& \supseteq \frac{\digamma(\varrho)+\digamma(\varsigma)}{2}\left[I_{\varrho+}^{\alpha} w(\varsigma)+I_{\varsigma^{-}}^{\alpha} w(\varrho)\right]
\end{aligned}
$$

for $\alpha>0$. 
Recently, Zhao et al. introduced the co-ordinated convexity of interval-valued functions and proved some new Hermite-Hadamard inclusions like the following.

Definition 4 ([53]) Let $\digamma: \Delta \subset \mathbb{R}^{2} \rightarrow \mathbb{R}_{\mathcal{I}}^{+}$be an interval-valued function such that $\digamma(\vartheta, s)=[\underline{\digamma}(\vartheta, s), \bar{\digamma}(\vartheta, s)]$. Then $\digamma=[\underline{\digamma}(\vartheta, s), \bar{\digamma}(\vartheta, s)]: \Delta \rightarrow \mathbb{R}_{\mathcal{I}}^{+}$is said to be a coordinated convex interval-valued function if the following inclusion holds:

$$
\begin{aligned}
& \digamma(\vartheta \xi+(1-\vartheta) \eta, s u+(1-s) w) \\
& \quad \supseteq \vartheta s \digamma(\xi, u)+\vartheta(1-s) \digamma(\xi, w)+s(1-\vartheta) \digamma(\eta, u)+(1-s)(1-\vartheta) \digamma(\eta, w)
\end{aligned}
$$

for all $(\xi, \eta),(u, w) \in \Delta$ and $s, \vartheta \in[0,1]$.

Lemma 1 ([53]) A function $\digamma=[\underline{\digamma}(\vartheta, s), \bar{\digamma}(\vartheta, s)]: \Delta \rightarrow \mathbb{R}_{\mathcal{I}}^{+}$is a co-ordinated convex interval-valued function if and only if there exist two functions $\digamma_{\xi}:[\zeta, l] \rightarrow \mathbb{R}_{\mathcal{I}}^{+}, \digamma_{\xi}(w)=$ $\digamma(\xi, w)$, and $\digamma_{\eta}:[\varrho, \varsigma] \rightarrow \mathbb{R}_{\mathcal{I}}^{+}, \digamma_{\eta}(u)=\digamma(u, \eta)$ are convex interval-valued functions.

Theorem 10 ([53]) Let $\digamma, \Omega: \Delta \rightarrow \mathbb{R}_{\mathcal{I}}^{+}$be two co-ordinated convex interval-valued functions such that $\digamma(\vartheta, s)=[\underline{\digamma}(\vartheta, s), \bar{\digamma}(\vartheta, s)]$ and $\Omega(\vartheta, s)=[\underline{\Omega}(\vartheta, s), \bar{\Omega}(\vartheta, s)]$, then the following Hermite-Hadamard type inclusions hold:

$$
\begin{aligned}
& \frac{1}{(\varsigma-\varrho)(\iota-\zeta)}(I R) \int_{\varrho}^{\varsigma} \int_{\zeta}^{\iota} \digamma(\xi, \eta) \Omega(\xi, \eta) d \eta d \xi \\
& \supseteq \frac{1}{9} \mathbf{K}(\varrho, \zeta, \zeta, \iota)+\frac{1}{18}[\mathbf{L}(\varrho, \zeta, \zeta, \iota)+\mathbf{M}(\varrho, \varsigma, \zeta, \iota)]+\frac{1}{36} \mathbf{N}(\varrho, \varsigma, \zeta, \iota)
\end{aligned}
$$

and

$$
\begin{aligned}
4 \digamma & \left(\frac{\varrho+\zeta}{2}, \frac{\zeta+\iota}{2}\right) \Omega\left(\frac{\varrho+\zeta}{2}, \frac{\zeta+\iota}{2}\right) \\
\supseteq & \frac{1}{(\varsigma-\varrho)(\iota-\zeta)}(I R) \int_{\varrho}^{\zeta} \int_{\zeta}^{\iota} \digamma(\xi, \eta) \Omega(\xi, \eta) d \eta d \xi+\frac{5}{36} \mathbf{K}(\varrho, \zeta, \zeta, \iota) \\
& +\frac{7}{36}[\mathbf{L}(\varrho, \zeta, \zeta, \iota)+\mathbf{M}(\varrho, \zeta, \zeta, \iota)]+\frac{2}{9} \mathbf{N}(\varrho, \zeta, \zeta, \iota),
\end{aligned}
$$

where

$$
\begin{aligned}
& \mathbf{K}(\varrho, \zeta, \zeta, \iota)=\digamma(\varrho, \zeta) \Omega(\varrho, \zeta)+\digamma(\zeta, \zeta) \Omega(\zeta, \zeta)+\digamma(\varrho, \iota) \Omega(\varrho, \iota)+\digamma(\zeta, \iota) \Omega(\zeta, \iota), \\
& \mathbf{L}(\varrho, \zeta, \zeta, \iota)=\digamma(\varrho, \zeta) \Omega(\varsigma, \zeta)+\digamma(\zeta, \zeta) \Omega(\varrho, \zeta)+\digamma(\varrho, \iota) \Omega(\varsigma, \iota)+\digamma(\varsigma, \iota) \Omega(\varrho, \iota), \\
& \mathbf{M}(\varrho, \zeta, \zeta, \iota)=\digamma(\varrho, \zeta) \Omega(\varrho, \iota)+\digamma(\zeta, \zeta) \Omega(\varsigma, \iota)+\digamma(\varrho, \iota) \Omega(\varrho, \zeta)+\digamma(\zeta, \iota) \Omega(\varsigma, \zeta),
\end{aligned}
$$

and

$$
\mathbf{N}(\varrho, \zeta, \zeta, \iota)=\digamma(\varrho, \zeta) \Omega(\varsigma, \iota)+\digamma(\zeta, \zeta) \Omega(\varrho, \iota)+\digamma(\varrho, \iota) \Omega(\zeta, \zeta)+\digamma(\zeta, \iota) \Omega(\varrho, \zeta) .
$$

For more recent results related to inclusions (2.6) and (2.7), one can read [23]. 
The definitions of the interval-valued Riemann-Liouville fractional integrals of function $\digamma(\xi, \eta)$ are given as follows.

Definition $5([4,23])$ Let $\digamma: \Delta \subset \mathbb{R}^{2} \rightarrow \mathbb{R}_{\mathcal{I}}^{+}$be an interval-valued function such that $\digamma(\vartheta, s)=[\underline{\digamma}(\vartheta, s), \bar{\digamma}(\vartheta, s)]$ and $\digamma \in \mathcal{I R}_{([\rho, \zeta])}$. The Riemann-Liouville fractional integrals $\mathcal{J}_{\varrho^{+, \zeta+}}^{\alpha, \beta}, \mathcal{J}_{\varrho^{+,}, l-}^{\alpha, \beta}, \mathcal{J}_{\zeta^{-, \zeta+}}^{\alpha, \beta}$ and $\mathcal{J}_{\zeta^{-, l-}}^{\alpha, \beta}$ of order $\alpha, \beta>0$ with $\varrho, \zeta \geq 0$ are defined by

$$
\begin{array}{ll}
\mathcal{J}_{\varrho+, \zeta+}^{\alpha, \beta} \digamma(\xi, \eta)=\frac{1}{\Gamma(\alpha) \Gamma(\beta)}(I R) \int_{\varrho}^{\xi} \int_{\zeta}^{\eta}(\xi-\vartheta)^{\alpha-1}(\eta-s)^{\beta-1} \digamma(\vartheta, s) d s d \vartheta, & \xi>\varrho, \eta>\zeta, \\
\mathcal{J}_{\varrho^{+, \ell-}}^{\alpha, \beta} \digamma(\xi, \eta)=\frac{1}{\Gamma(\alpha) \Gamma(\beta)}(I R) \int_{\varrho}^{\xi} \int_{\eta}^{\iota}(\xi-\vartheta)^{\alpha-1}(s-\eta)^{\beta-1} \digamma(\vartheta, s) d s d \vartheta, & \xi>\varrho, \eta>\iota, \\
\mathcal{J}_{\zeta^{-, \zeta+}}^{\alpha, \beta} \digamma(\xi, \eta)=\frac{1}{\Gamma(\alpha) \Gamma(\beta)}(I R) \int_{\xi}^{\zeta} \int_{\zeta}^{\eta}(\vartheta-\xi)^{\alpha-1}(\eta-s)^{\beta-1} \digamma(\vartheta, s) d s d \vartheta, & \xi<\zeta, \eta>\zeta, \\
\mathcal{J}_{\varsigma^{-,, l}}^{\alpha, \beta} \digamma(\xi, \eta)=\frac{1}{\Gamma(\alpha) \Gamma(\beta)}(I R) \int_{\xi}^{\zeta} \int_{\eta}^{\iota}(\vartheta-\xi)^{\alpha-1}(s-\eta)^{\beta-1} \digamma(\vartheta, s) d s d \vartheta, & \xi<\zeta, \eta<\iota,
\end{array}
$$

respectively.

\section{Main results}

Theorem 11 Let $\digamma, \Omega: \Delta \subset \mathbb{R}^{2} \rightarrow \mathbb{R}_{\mathcal{I}}^{+}$be two co-ordinated convex interval-valued functions on $\Delta$ such that $\digamma(\vartheta, s)=[\underline{\digamma}(\vartheta, s), \bar{\digamma}(\vartheta, s)]$ and $\Omega(\vartheta, s)=[\underline{\Omega}(\vartheta, s), \bar{\Omega}(\vartheta, s)]$. In addition, $w_{1}:[\varrho, \varsigma] \rightarrow \mathbb{R}$ is nonnegative, integrable, and symmetric about $\xi=\frac{\varrho+\zeta}{2}\left(\right.$ i.e. $w_{1}(\xi)=$ $\left.w_{1}(\varrho+\varsigma-\xi)\right)$ and $w_{2}:[\zeta, l] \rightarrow \mathbb{R}$ is nonnegative, integrable, and symmetric about $\eta=\frac{\zeta+\iota}{2}$. Then we have the following Hermite-Hadamard-Fejer type inclusion:

$$
\begin{aligned}
& \frac{1}{(\varsigma-\varrho)(\iota-\zeta)}(I R) \int_{\varrho}^{\varsigma} \int_{\zeta}^{\iota} \digamma(\xi, \eta) \Omega(\xi, \eta) w_{1}(\xi) w_{2}(\eta) d \eta d \xi \\
& \supseteq \frac{\mathcal{A}_{2}(\eta ; \zeta, \iota)}{(\varsigma-\varrho)^{3}(\iota-\zeta)^{3}}\left[\mathbf{K}(\varrho, \varsigma, \zeta, \iota) \mathcal{A}_{1}(\xi ; \varrho, \varsigma)+\mathbf{L}(\varrho, \varsigma, \zeta, \iota) \mathcal{B}_{1}(\xi ; \varrho, \varsigma)\right] \\
& \quad+\frac{\mathcal{B}_{2}(\eta ; \zeta, \iota)}{(\varsigma-\varrho)^{3}(\iota-\zeta)^{3}}\left[\mathbf{M}(\varrho, \varsigma, \zeta, \iota) \mathcal{A}_{1}(\xi ; \varrho, \varsigma)+\mathbf{N}(\varrho, \varsigma, \zeta, \iota) \mathcal{B}_{1}(\xi ; \varrho, \varsigma)\right],
\end{aligned}
$$

where $\mathbf{K}(\varrho, \varsigma, \zeta, \iota), \mathbf{L}(\varrho, \varsigma, \zeta, \iota), \mathbf{M}(\varrho, \zeta, \zeta, \iota)$, and $\mathbf{N}(\varrho, \zeta, \zeta, \iota)$ defined as in Theorem 10.

Proof Since $\digamma$ and $\Omega$ are two co-ordinated convex interval-valued functions on $\Delta$, the functions $\digamma_{\xi}(\eta)=\digamma(\xi, \eta)$ and $\Omega_{\xi}(\eta)=\Omega(\xi, \eta)$ are convex interval-valued on $[\zeta, \iota]$. If inclusion (2.3) is applied to the functions $\digamma_{\xi}$ and $\Omega_{\xi}$, then we obtain

$$
\begin{aligned}
& \frac{1}{\iota-\zeta}(I R) \int_{\zeta}^{\iota} \digamma_{\xi}(\eta) \Omega_{\xi}(\eta) w_{2}(\eta) d \eta \\
& \supseteq \frac{\mathcal{A}_{2}(\eta ; \zeta, \iota)}{(\iota-\zeta)^{3}}\left[\digamma_{\xi}(\zeta) \Omega_{\xi}(\zeta)+\digamma_{\xi}(\iota) \Omega_{\xi}(\iota)\right] \\
& \quad+\frac{\mathcal{B}_{2}(\eta ; \zeta, \iota)}{(\iota-\zeta)^{3}}\left[\digamma_{\xi}(\zeta) \Omega_{\xi}(\iota)+\digamma_{\xi}(\iota) \Omega_{\xi}(\zeta)\right] .
\end{aligned}
$$


This implies that

$$
\begin{aligned}
& \frac{1}{\iota-\zeta}(I R) \int_{\zeta}^{\iota} \digamma(\xi, \eta) \Omega(\xi, \eta) w_{2}(\eta) d \eta \\
& \supseteq \frac{\mathcal{A}_{2}(\eta ; \zeta, \iota)}{(\iota-\zeta)^{3}}[\digamma(\xi, \zeta) \Omega(\xi, \zeta)+\digamma(\xi, \iota) \Omega(\xi, \iota)] \\
& \quad+\frac{\mathcal{B}_{2}(\eta ; \zeta, \iota)}{(\iota-\zeta)^{3}}[\digamma(\xi, \zeta) \Omega(\xi, \iota)+\digamma(\xi, \iota) \Omega(\xi, \zeta)] .
\end{aligned}
$$

Multiplying (3.2) by $\frac{w_{1}(\xi)}{\varsigma-\varrho}$ and integrating the resultant one with respect to $\xi$ over $[\varrho, \varsigma]$, we get

$$
\begin{aligned}
& \frac{1}{(\varsigma-\varrho)(\iota-\zeta)}(I R) \int_{\varrho}^{\varsigma} \int_{\zeta}^{\iota} \digamma(\xi, \eta) \Omega(\xi, \eta) w_{1}(\xi) w_{2}(\eta) d \eta d \xi \\
& \supseteq \frac{\mathcal{A}_{2}(\eta ; \zeta, \iota)}{(\varsigma-\varrho)(\iota-\zeta)^{3}}(I R) \int_{\varrho}^{\zeta}[\digamma(\xi, \zeta) \Omega(\xi, \zeta)+\digamma(\xi, \iota) \Omega(\xi, \iota)] w_{1}(\xi) d \xi \\
& \quad+\frac{\mathcal{B}_{2}(\eta ; \zeta, \iota)}{(\varsigma-\varrho)(\iota-\zeta)^{3}}(I R) \int_{\varrho}^{\varsigma}[\digamma(\xi, \zeta) \Omega(\xi, \iota)+\digamma(\xi, \iota) \Omega(\xi, \zeta)] w_{1}(\xi) d \xi
\end{aligned}
$$

Applying (2.3) to each integral in (3.3), we have

$$
\begin{aligned}
& (I R) \int_{\varrho}^{\zeta} \digamma(\xi, \zeta) \Omega(\xi, \zeta) w_{1}(\xi) d \xi \\
& \supseteq \frac{\mathcal{A}_{1}(\xi ; \varrho, \varsigma)}{(\varsigma-\varrho)^{2}}[\digamma(\varrho, \zeta) \Omega(\varrho, \zeta)+\digamma(\varsigma, \zeta) \Omega(\varsigma, \zeta)] \\
& +\frac{\mathcal{B}_{1}(\xi ; \varrho, \varsigma)}{(\varsigma-\varrho)^{2}}[\digamma(\varrho, \zeta) \Omega(\varsigma, \zeta)+\digamma(\varsigma, \zeta) \Omega(\varrho, \zeta)] \\
& (I R) \int_{\varrho}^{\varsigma} \digamma(\xi, \iota) \Omega(\xi, \iota) w_{1}(\xi) d \xi \\
& \supseteq \frac{\mathcal{A}_{1}(\xi ; \varrho, \varsigma)}{(\varsigma-\varrho)^{2}}[\digamma(\varrho, \iota) \Omega(\varrho, \iota)+\digamma(\varsigma, \iota) \Omega(\varsigma, \iota)] \\
& +\frac{\mathcal{B}_{1}(\xi ; \varrho, \varsigma)}{(\varsigma-\varrho)^{2}}[\digamma(\varrho, \iota) \Omega(\varsigma, \iota)+\digamma(\varsigma, \iota) \Omega(\varrho, \iota)], \\
& (I R) \int_{\varrho}^{\varsigma} \digamma(\xi, \zeta) \Omega(\xi, \iota) w_{1}(\xi) d \xi \\
& \supseteq \frac{\mathcal{A}_{1}(\xi ; \varrho, \varsigma)}{(\varsigma-\varrho)^{2}}[\digamma(\varrho, \zeta) \Omega(\varrho, \iota)+\digamma(\varsigma, \zeta) \Omega(\varsigma, \iota)] \\
& +\frac{\mathcal{B}_{1}(\xi ; \varrho, \varsigma)}{(\varsigma-\varrho)^{2}}[\digamma(\varrho, \zeta) \Omega(\varsigma, \iota)+\digamma(\varsigma, \zeta) \Omega(\varrho, \iota)],
\end{aligned}
$$

and

$$
(I R) \int_{\varrho}^{\varsigma} \digamma(\xi, \iota) \Omega(\xi, \zeta) w_{1}(\xi) d \xi
$$




$$
\begin{aligned}
\supseteq & \frac{\mathcal{A}_{1}(\xi ; \varrho, \varsigma)}{(\varsigma-\varrho)^{2}}[\digamma(\varrho, \iota) \Omega(\varrho, \zeta)+\digamma(\varsigma, \iota) \Omega(\varsigma, \zeta)] \\
& +\frac{\mathcal{B}_{1}(\xi ; \varrho, \zeta)}{(\varsigma-\varrho)^{2}}[\digamma(\varrho, \iota) \Omega(\varsigma, \zeta)+\digamma(\varsigma, \iota) \Omega(\varrho, \zeta)] .
\end{aligned}
$$

Substituting (3.4)-(3.7) in (3.3) and by arranging the resultant one, we get the desired result. On the other hand, the same result can be obtained by using the convexity of intervalvalued functions $\digamma_{\eta}(\xi)=\digamma(\xi, \eta)$ and $\Omega_{\eta}(\xi)=\Omega(\xi, \eta)$.

Theorem 12 Let $\digamma, \Omega: \Delta \subset \mathbb{R}^{2} \rightarrow \mathbb{R}_{\mathcal{I}}^{+}$be two co-ordinated convex interval-valued functions on $\Delta$ such that $\digamma(\vartheta, s)=[\underline{\digamma}(\vartheta, s), \bar{\digamma}(\vartheta, s)]$ and $\Omega(\vartheta, s)=[\underline{\Omega}(\vartheta, s), \bar{\Omega}(\vartheta, s)]$. In addition, $w_{1}:[\varrho, \varsigma] \rightarrow \mathbb{R}$ is nonnegative, integrable, and symmetric about $\xi=\frac{\varrho+\varsigma}{2}\left(\right.$ i.e. $w_{1}(\xi)=$ $\left.w_{1}(\varrho+\varsigma-\xi)\right)$ and $w_{2}:[\zeta, l] \rightarrow \mathbb{R}$ is nonnegative, integrable, and symmetric about $\eta=\frac{\zeta+l}{2}$. Then we have the following Hermite-Hadamard-Fejér type inclusion:

$$
\begin{aligned}
4(I R) & \int_{\varrho}^{\varsigma} \int_{\zeta}^{\iota} F\left(\frac{\varrho+\varsigma}{2}, \frac{\zeta+\iota}{2}\right) \Omega\left(\frac{\varrho+\varsigma}{2}, \frac{\zeta+\iota}{2}\right) w_{1}(\xi) w_{2}(\eta) d \eta d \xi \\
\supseteq & (I R) \int_{\varrho}^{\varsigma} \int_{\zeta}^{\iota} \digamma(\xi, \eta) \Omega(\xi, \eta) w_{1}(\xi) w_{2}(\eta) d \eta d \xi \\
& +\frac{\mathbf{K}(\varrho, \varsigma, \zeta, \iota)}{(\varsigma-\varrho)^{2}(\iota-\zeta)^{2}}\left[\mathcal{B}_{1}(\xi ; \varrho, \varsigma) \mathcal{A}_{2}(\eta ; \zeta, \iota)+\mathcal{B}_{2}(\eta ; \zeta, \iota) \mathcal{A}_{1}(\xi ; \varrho, \varsigma)\right. \\
& \left.+\mathcal{B}_{1}(\xi ; \varrho, \varsigma) \mathcal{B}_{2}(\eta ; \zeta, \iota)\right] \\
& +\frac{\mathbf{L}(\varrho, \varsigma, \zeta, \iota)}{(\varsigma-\varrho)^{2}(\iota-\zeta)^{2}}\left[\mathcal{B}_{2}(\eta ; \zeta, \iota) \mathcal{B}_{1}(\xi ; \varrho, \varsigma)+\mathcal{A}_{2}(\eta ; \zeta, \iota) \mathcal{A}_{1}(\xi ; \varrho, \varsigma)\right. \\
& \left.+\mathcal{A}_{1}(\xi ; \varrho, \varsigma) \mathcal{B}_{2}(\eta ; \zeta, \iota)\right] \\
& +\frac{\mathbf{M}(\varrho, \varsigma, \zeta, \iota)}{(\varsigma-\varrho)^{2}(\iota-\zeta)^{2}}\left[\mathcal{B}_{2}(\eta ; \zeta, \iota) \mathcal{B}_{1}(\xi ; \varrho, \varsigma)+\mathcal{A}_{2}(\eta ; \zeta, \iota) \mathcal{A}_{1}(\xi ; \varrho, \varsigma)\right. \\
& \left.+\mathcal{B}_{1}(\xi ; \varrho, \varsigma) \mathcal{A}_{2}(\eta ; \zeta, \iota)\right] \\
& +\frac{\mathbf{N}(\varrho, \varsigma, \zeta, \iota)}{(\varsigma-\varrho)^{2}(\iota-\zeta)^{2}}\left[\mathcal{A}_{1}(\xi ; \varrho, \varsigma) \mathcal{B}_{2}(\eta ; \zeta, \iota)+\mathcal{A}_{2}(\eta ; \zeta, \iota) \mathcal{B}_{1}(\xi ; \varrho, \varsigma)\right. \\
& \left.+\mathcal{A}_{2}(\eta ; \zeta, \iota) \mathcal{A}_{1}(\xi ; \varrho, \varsigma)\right] .
\end{aligned}
$$

Proof Since $\digamma$ and $\Omega$ are co-ordinated convex interval-valued functions on $\Delta$, the functions $\digamma_{\xi}, \Omega_{\xi}, \digamma_{\eta}$, and $\Omega_{\eta}$ are convex interval-valued. Applying (2.4) for the functions $\digamma\left(\xi, \frac{\zeta+\iota}{2}\right)$ and $\Omega\left(\xi, \frac{\zeta+l}{2}\right)$ and multiplying the resultant by $2 \int_{\zeta}^{\iota} w_{2}(\eta) d \eta$, we get

$$
\begin{aligned}
4 \digamma\left(\frac{\varrho+\varsigma}{2}, \frac{\zeta+\iota}{2}\right) \Omega\left(\frac{\varrho+\varsigma}{2}, \frac{\zeta+\iota}{2}\right) \int_{\varrho}^{\varsigma} \int_{\zeta}^{\iota} w_{1}(\xi) w_{2}(\eta) d \eta d \xi \\
\supseteq 2(I R) \int_{\varrho}^{\varsigma} \int_{\zeta}^{\iota} \digamma\left(\xi, \frac{\zeta+\iota}{2}\right) \Omega\left(\xi, \frac{\zeta+\iota}{2}\right) w_{1}(\xi) w_{2}(\eta) d \eta d \xi \\
\quad+\frac{2}{(\varsigma-\varrho)^{2}}\left[\digamma\left(\varrho, \frac{\zeta+\iota}{2}\right) \Omega\left(\varrho, \frac{\zeta+\iota}{2}\right)+\digamma\left(\varsigma, \frac{\zeta+\iota}{2}\right) \Omega\left(\varsigma, \frac{\zeta+\iota}{2}\right)\right] \\
\quad \times\left(\int_{\zeta}^{\iota} w_{2}(\eta) d \eta\right) \mathcal{B}_{1}(\xi ; \varrho, \zeta)
\end{aligned}
$$




$$
\begin{aligned}
& +\frac{2}{(\varsigma-\varrho)^{2}}\left[\digamma\left(\varrho, \frac{\zeta+\iota}{2}\right) \Omega\left(\varsigma, \frac{\zeta+\iota}{2}\right)+\digamma\left(\varsigma, \frac{\zeta+\iota}{2}\right) \Omega\left(\varrho, \frac{\zeta+\iota}{2}\right)\right] \\
& \times\left(\int_{\zeta}^{\iota} w_{2}(\eta) d \eta\right) \mathcal{A}_{1}(\xi ; \varrho, \varsigma) .
\end{aligned}
$$

Similarly, if we apply (2.4) for the functions $\digamma\left(\frac{\varrho+\zeta}{2}, \eta\right)$ and $\Omega\left(\frac{\varrho+\zeta}{2}, \eta\right)$ and multiply the resultant inclusion by $2 \int_{\varrho}^{\varsigma} w_{1}(\xi) d \xi$, we get

$$
\begin{aligned}
4 \digamma\left(\frac{\varrho+\zeta}{2}, \frac{\zeta+\iota}{2}\right) \Omega\left(\frac{\varrho+\zeta}{2}, \frac{\zeta+\iota}{2}\right) \int_{\varrho}^{\zeta} \int_{\zeta}^{\iota} w_{1}(\xi) w_{2}(\eta) d \eta d \xi \\
\supseteq 2(I R) \int_{\varrho}^{\zeta} \int_{\zeta}^{\iota} \digamma\left(\frac{\varrho+\zeta}{2}, \eta\right) \Omega\left(\frac{\varrho+\zeta}{2}, \eta\right) w_{1}(\xi) w_{2}(\eta) d \eta d \xi \\
\quad+\frac{2}{(\iota-\zeta)^{2}}\left[\digamma\left(\frac{\varrho+\zeta}{2}, \zeta\right) \Omega\left(\frac{\varrho+\zeta}{2}, \zeta\right)+\digamma\left(\frac{\varrho+\zeta}{2}, \iota\right) \Omega\left(\frac{\varrho+\zeta}{2}, \iota\right)\right] \\
\quad \times\left(\int_{\varrho}^{\zeta} w_{1}(\xi) d \xi\right) \mathcal{B}_{2}(\eta ; \zeta, \iota) \\
\quad+\frac{2}{(\iota-\zeta)^{2}}\left[\digamma\left(\frac{\varrho+\zeta}{2}, \zeta\right) \Omega\left(\frac{\varrho+\zeta}{2}, \iota\right)+\digamma\left(\frac{\varrho+\zeta}{2}, \iota\right) \Omega\left(\frac{\varrho+\zeta}{2}, \zeta\right)\right] \\
\quad \times\left(\int_{\varrho}^{\zeta} w_{1}(\xi) d \xi\right) \mathcal{A}_{2}(\eta ; \zeta, \iota) .
\end{aligned}
$$

Using (2.4) for each term in the right-hand sides of (3.8) and (3.9), we have

$$
\begin{aligned}
& 2 \digamma\left(\varrho, \frac{\zeta+\iota}{2}\right) \Omega\left(\varrho, \frac{\zeta+\iota}{2}\right) \int_{\zeta}^{\iota} w_{2}(\eta) d \eta \\
& \supseteq(I R) \int_{\zeta}^{\iota} \digamma(\varrho, \eta) \Omega(\varrho, \eta) w_{2}(\eta) d \eta \\
& +\left[\frac{\digamma(\varrho, \zeta) \Omega(\varrho, \zeta)+\digamma(\varrho, \iota) \Omega(\varrho, \iota)}{(\iota-\zeta)^{2}}\right] \mathcal{B}_{1}(\eta ; \zeta, \iota) \\
& +\left[\frac{\digamma(\varrho, \zeta) \Omega(\varrho, \iota)+\digamma(\varrho, \iota) \Omega(\varrho, \zeta)}{(\iota-\zeta)^{2}}\right] \mathcal{A}_{1}(\eta ; \zeta, \iota), \\
& 2 \digamma\left(\varsigma, \frac{\zeta+\iota}{2}\right) \Omega\left(\varsigma, \frac{\zeta+\iota}{2}\right) \int_{\zeta}^{\iota} w_{2}(\eta) d \eta \\
& \supseteq(I R) \int_{\zeta}^{\iota} \digamma(\varsigma, \eta) \Omega(\varsigma, \eta) w_{2}(\eta) d \eta \\
& +\left[\frac{\digamma(\zeta, \zeta) \Omega(\zeta, \zeta)+\digamma(\zeta, \iota) \Omega(\zeta, \iota)}{(\iota-\zeta)^{2}}\right] \mathcal{B}_{1}(\eta ; \zeta, \iota) \\
& +\left[\frac{\digamma(\zeta, \zeta) \Omega(\zeta, \iota)+\digamma(\zeta, \iota) \Omega(\zeta, \zeta)}{(\iota-\zeta)^{2}}\right] \mathcal{A}_{1}(\eta ; \zeta, \iota), \\
& 2 \digamma\left(\varrho, \frac{\zeta+\iota}{2}\right) \Omega\left(\varsigma, \frac{\zeta+\iota}{2}\right) \int_{\zeta}^{\iota} w_{2}(\eta) d \eta \\
& \supseteq(I R) \int_{\zeta}^{\iota} \digamma(\varrho, \eta) \Omega(\varsigma, \eta) w_{2}(\eta) d \eta \\
& +\left[\frac{\digamma(\varrho, \zeta) \Omega(\zeta, \zeta)+\digamma(\varrho, \iota) \Omega(\varsigma, \iota)}{(\iota-\zeta)^{2}}\right] \mathcal{B}_{1}(\eta ; \zeta, \iota)
\end{aligned}
$$




$$
\begin{aligned}
& +\left[\frac{\digamma(\varrho, \zeta) \Omega(\zeta, \iota)+\digamma(\varrho, \iota) \Omega(\varsigma, \zeta)}{(\iota-\zeta)^{2}}\right] \mathcal{A}_{1}(\eta ; \zeta, \iota), \\
& 2 \digamma\left(\varsigma, \frac{\zeta+\iota}{2}\right) \Omega\left(\varrho, \frac{\zeta+\iota}{2}\right) \int_{\zeta}^{\iota} w_{2}(\eta) d \eta \\
& \supseteq(I R) \int_{\zeta}^{\iota} \digamma(\varsigma, \eta) \Omega(\varrho, \eta) w_{2}(\eta) d \eta \\
& +\left[\frac{\digamma(\varsigma, \zeta) \Omega(\varrho, \zeta)+\digamma(\varsigma, \iota) \Omega(\varrho, \iota)}{(\iota-\zeta)^{2}}\right] \mathcal{B}_{1}(\eta ; \zeta, \iota) \\
& +\left[\frac{\digamma(\varsigma, \zeta) \Omega(\varrho, \iota)+\digamma(\varsigma, \iota) \Omega(\varrho, \zeta)}{(\iota-\zeta)^{2}}\right] \mathcal{A}_{1}(\eta ; \zeta, \iota), \\
& 2 \digamma\left(\frac{\varrho+\zeta}{2}, \zeta\right) \Omega\left(\frac{\varrho+\zeta}{2}, \zeta\right) \int_{\varrho}^{\zeta} w_{1}(\xi) d \xi \\
& \supseteq(I R) \int_{\varrho}^{\varsigma} \digamma(\xi, \zeta) \Omega(\xi, \zeta) w_{1}(\xi) d \xi \\
& +\left[\frac{\digamma(\varrho, \zeta) \Omega(\varrho, \zeta)+\digamma(\varsigma, \zeta) \Omega(\varsigma, \zeta)}{(\varsigma-\varrho)^{2}}\right] \mathcal{B}_{1}(\xi ; \varrho, \varsigma) \\
& +\left[\frac{\digamma(\varrho, \zeta) \Omega(\varsigma, \zeta)+\digamma(\varsigma, \zeta) \Omega(\varrho, \zeta)}{(\varsigma-\varrho)^{2}}\right] \mathcal{A}_{1}(\xi ; \varrho, \varsigma), \\
& 2 \digamma\left(\frac{\varrho+\varsigma}{2}, \iota\right) \Omega\left(\frac{\varrho+\varsigma}{2}, \iota\right) \int_{\varrho}^{\varsigma} w_{1}(\xi) d \xi \\
& \supseteq(I R) \int_{\varrho}^{\varsigma} \digamma(\xi, \iota) \Omega(\xi, \iota) w_{1}(\xi) d \xi \\
& +\left[\frac{\digamma(\varrho, \iota) \Omega(\varrho, \iota)+\digamma(\varsigma, \iota) \Omega(\varsigma, \iota)}{(\varsigma-\varrho)^{2}}\right] \mathcal{B}_{1}(\xi ; \varrho, \varsigma) \\
& +\left[\frac{\digamma(\varrho, \iota) \Omega(\varsigma, \iota)+\digamma(\varsigma, \iota) \Omega(\varrho, \iota)}{(\varsigma-\varrho)^{2}}\right] \mathcal{A}_{1}(\xi ; \varrho, \varsigma), \\
& 2 \digamma\left(\frac{\varrho+\varsigma}{2}, \iota\right) \Omega\left(\frac{\varrho+\varsigma}{2}, \iota\right) \int_{\varrho}^{\varsigma} w_{1}(\xi) d \xi \\
& \supseteq(I R) \int_{\varrho}^{\varsigma} \digamma(\xi, \iota) \Omega(\xi, \iota) w_{1}(\xi) d \xi \\
& +\left[\frac{\digamma(\varrho, \iota) \Omega(\varrho, \iota)+\digamma(\varsigma, \iota) \Omega(\varsigma, \iota)}{(\varsigma-\varrho)^{2}}\right] \mathcal{B}_{1}(\xi ; \varrho, \varsigma) \\
& +\left[\frac{\digamma(\varrho, \iota) \Omega(\varsigma, \iota)+\digamma(\varsigma, \iota) \Omega(\varrho, \iota)}{(\varsigma-\varrho)^{2}}\right] \mathcal{A}_{1}(\xi ; \varrho, \varsigma), \\
& 2 \digamma\left(\frac{\varrho+\varsigma}{2}, \zeta\right) \Omega\left(\frac{\varrho+\zeta}{2}, \iota\right) \int_{\varrho}^{\varsigma} w_{1}(\xi) d \xi \\
& \supseteq(I R) \int_{\varrho}^{\varsigma} \digamma(\xi, \zeta) \Omega(\xi, \iota) w_{1}(\xi) d \xi \\
& +\left[\frac{\digamma(\varrho, \zeta) \Omega(\varrho, \iota)+\digamma(\zeta, \zeta) \Omega(\varsigma, \iota)}{(\varsigma-\varrho)^{2}}\right] \mathcal{B}_{1}(\xi ; \varrho, \varsigma) \\
& +\left[\frac{\digamma(\varrho, \zeta) \Omega(\varsigma, \iota)+\digamma(\varsigma, \zeta) \Omega(\varrho, \iota)}{(\varsigma-\varrho)^{2}}\right] \mathcal{A}_{1}(\xi ; \varrho, \varsigma),
\end{aligned}
$$




$$
\begin{aligned}
2 \digamma\left(\frac{\varrho+\varsigma}{2}, \iota\right) \Omega\left(\frac{\varrho+\varsigma}{2}, \zeta\right) \int_{\varrho}^{\varsigma} w_{1}(\xi) d \xi \\
\supseteq(I R) \int_{\varrho}^{\varsigma} \digamma(\xi, \iota) \Omega(\xi, \zeta) w_{1}(\xi) d \xi \\
\quad+\left[\frac{\digamma(\varrho, \iota) \Omega(\varrho, \zeta)+\digamma(\varsigma, \iota) \Omega(\varsigma, \zeta)}{(\varsigma-\varrho)^{2}}\right] \mathcal{B}_{1}(\xi ; \varrho, \varsigma) \\
\quad+\left[\frac{\digamma(\varrho, \iota) \Omega(\varsigma, \zeta)+\digamma(\varsigma, \iota) \Omega(\varrho, \zeta)}{(\varsigma-\varrho)^{2}}\right] \mathcal{A}_{1}(\xi ; \varrho, \varsigma) .
\end{aligned}
$$

Putting (3.10)-(3.18) in (3.8) and (3.9) and adding the resultant one, we obtain

$$
\begin{aligned}
& 8 \digamma\left(\frac{\varrho+\zeta}{2}, \frac{\zeta+\iota}{2}\right) \Omega\left(\frac{\varrho+\zeta}{2}, \frac{\zeta+\iota}{2}\right) \int_{\varrho}^{\zeta} \int_{\zeta}^{\iota} w_{1}(\xi) w_{2}(\eta) d \eta d \xi \\
& \supseteq 2(I R) \int_{\varrho}^{\varsigma} \int_{\zeta}^{\iota} \digamma\left(\xi, \frac{\zeta+\iota}{2}\right) \Omega\left(\xi, \frac{\zeta+\iota}{2}\right) w_{1}(\xi) w_{2}(\eta) d \eta d \xi \\
& +2(I R) \int_{\varrho}^{\varsigma} \int_{\zeta}^{\iota} \digamma\left(\frac{\varrho+\varsigma}{2}, \eta\right) \Omega\left(\frac{\varrho+\varsigma}{2}, \eta\right) w_{1}(\xi) w_{2}(\eta) d \eta d \xi \\
& +\frac{\mathcal{B}_{1}(\xi ; \varrho, \zeta)}{(\varsigma-\varrho)^{2}}(I R) \int_{\zeta}^{\iota}[\digamma(\varrho, \eta) \Omega(\varrho, \eta)+\digamma(\varsigma, \eta) \Omega(\varsigma, \eta)] w_{2}(\eta) d \eta \\
& +\frac{\mathcal{A}_{1}(\xi ; \varrho, \varsigma)}{(\varsigma-\varrho)^{2}}(I R) \int_{\zeta}^{\iota}[\digamma(\varrho, \eta) \Omega(\varsigma, \eta)+\digamma(\varsigma, \eta) \Omega(\varrho, \eta)] w_{2}(\eta) d \eta \\
& +\frac{\mathcal{B}_{2}(\eta ; \zeta, \iota)}{(\iota-\zeta)^{2}}(I R) \int_{\varrho}^{\zeta}[\digamma(\xi, \zeta) \Omega(\xi, \zeta)+\digamma(\xi, \iota) \Omega(\xi, \iota)] w_{1}(\xi) d \xi \\
& +\frac{\mathcal{A}_{2}(\eta ; \zeta, \iota)}{(\iota-\zeta)^{2}}(I R) \int_{\varrho}^{\zeta}[\digamma(\xi, \zeta) \Omega(\xi, \iota)+\digamma(\xi, \iota) \Omega(\xi, \zeta)] w_{1}(\xi) d \xi \\
& +\frac{2 \mathbf{K}(\varrho, \varsigma, \zeta, \iota)}{(\varsigma-\varrho)^{2}(\iota-\zeta)^{2}} \mathcal{B}_{1}(\xi ; \varrho, \varsigma) \mathcal{B}_{2}(\eta ; \zeta, \iota) \\
& +\frac{2 \mathbf{L}(\varrho, \varsigma, \zeta, \iota)}{(\varsigma-\varrho)^{2}(\iota-\zeta)^{2}} \mathcal{A}_{1}(\xi ; \varrho, \varsigma) \mathcal{B}_{2}(\eta ; \zeta, \iota) \\
& +\frac{2 \mathbf{M}(\varrho, \zeta, \zeta, \iota)}{(\varsigma-\varrho)^{2}(\iota-\zeta)^{2}} \mathcal{B}_{1}(\xi ; \varrho, \varsigma) \mathcal{A}_{2}(\eta ; \zeta, \iota) \\
& +\frac{2 \mathbf{N}(\varrho, \varsigma, \zeta, \iota)}{(\varsigma-\varrho)^{2}(\iota-\zeta)^{2}} \mathcal{A}_{1}(\xi ; \varrho, \varsigma) \mathcal{A}_{2}(\eta ; \zeta, \iota)
\end{aligned}
$$

By applying (2.4) to the functions $\digamma\left(\xi, \frac{\zeta+\iota}{2}\right)$ and $\Omega\left(\xi, \frac{\zeta+\iota}{2}\right)$, we get

$$
\begin{aligned}
2(I R) & \int_{\varrho}^{\zeta} \int_{\zeta}^{\iota} \digamma\left(\xi, \frac{\zeta+\iota}{2}\right) \Omega\left(\xi, \frac{\zeta+\iota}{2}\right) w_{1}(\xi) w_{2}(\eta) d \eta d \xi \\
\supseteq & (I R) \int_{\varrho}^{\zeta} \int_{\zeta}^{\iota} \digamma(\xi, \eta) \Omega(\xi, \eta) w_{1}(\xi) w_{2}(\eta) d \eta d \xi \\
& +\frac{\mathcal{B}_{2}(\eta ; \zeta, \iota)}{(\iota-\zeta)^{2}}(I R) \int_{\varrho}^{\zeta}[\digamma(\xi, \zeta) \Omega(\xi, \zeta)+\digamma(\xi, \iota) \Omega(\xi, \iota)] w_{1}(\xi) d \xi \\
& +\frac{\mathcal{A}_{2}(\eta ; \zeta, \iota)}{(\iota-\zeta)^{2}}(I R) \int_{\varrho}^{\zeta}[\digamma(\xi, \zeta) \Omega(\xi, \iota)+\digamma(\xi, \iota) \Omega(\xi, \zeta)] w_{1}(\xi) d \xi
\end{aligned}
$$


Similarly, if we apply $(2.4)$ to the functions $\digamma\left(\frac{\varrho+\varsigma}{2}, \eta\right)$ and $\Omega\left(\frac{\varrho+\varsigma}{2}, \eta\right)$, then we have

$$
\begin{aligned}
& 2(I R) \int_{\varrho}^{\varsigma} \int_{\zeta}^{\iota} \digamma\left(\frac{\varrho+\varsigma}{2}, \eta\right) \Omega\left(\frac{\varrho+\varsigma}{2}, \eta\right) w_{1}(\xi) w_{2}(\eta) d \eta d \xi \\
& \supseteq(I R) \int_{\varrho}^{\varsigma} \int_{\zeta}^{\iota} \digamma(\xi, \eta) \Omega(\xi, \eta) w_{1}(\xi) w_{2}(\eta) d \eta d \xi \\
&+\frac{\mathcal{B}_{1}(\xi ; \varrho, \varsigma)}{(\varsigma-\varrho)^{2}}(I R) \int_{\zeta}^{\iota}[\digamma(\varrho, \eta) \Omega(\varrho, \eta)+\digamma(\varsigma, \eta) \Omega(\varsigma, \eta)] w_{2}(\eta) d \eta \\
&+\frac{\mathcal{A}_{1}(\xi ; \varrho, \varsigma)}{(\varsigma-\varrho)^{2}}(I R) \int_{\zeta}^{\iota}[\digamma(\varrho, \eta) \Omega(\varsigma, \eta)+\digamma(\varsigma, \eta) \Omega(\varrho, \eta)] w_{2}(\eta) d \eta .
\end{aligned}
$$

Substituting (3.20) and (3.21) in (3.19), we have

$$
\begin{aligned}
& 8 \digamma\left(\frac{\varrho+\zeta}{2}, \frac{\zeta+\iota}{2}\right) \Omega\left(\frac{\varrho+\zeta}{2}, \frac{\zeta+\iota}{2}\right) \int_{\varrho}^{\zeta} \int_{\zeta}^{\iota} w_{1}(\xi) w_{2}(\eta) d \eta d \xi \\
& \supseteq 2(I R) \int_{\varrho}^{\varsigma} \int_{\zeta}^{\iota} \digamma(\xi, \eta) \Omega(\xi, \eta) w_{1}(\xi) w_{2}(\eta) d \eta d \xi \\
& +\frac{2 B_{1}(\xi ; \varrho, \varsigma)}{(\varsigma-\varrho)^{2}}(I R) \int_{\zeta}^{\iota}[\digamma(\varrho, \eta) \Omega(\varrho, \eta)+\digamma(\varsigma, \eta) \Omega(\varsigma, \eta)] w_{2}(\eta) d \eta \\
& +\frac{2 A_{1}(\xi ; \varrho, \varsigma)}{(\varsigma-\varrho)^{2}}(I R) \int_{\zeta}^{\iota}[\digamma(\varrho, \eta) \Omega(\varsigma, \eta)+\digamma(\varsigma, \eta) \Omega(\varrho, \eta)] w_{2}(\eta) d \eta \\
& +\frac{2 B_{2}(\eta ; \zeta, \iota)}{(\iota-\zeta)^{2}}(I R) \int_{\varrho}^{\zeta}[\digamma(\xi, \zeta) \Omega(\xi, \zeta)+\digamma(\xi, \iota) \Omega(\xi, \iota)] w_{1}(\xi) d \xi \\
& +\frac{2 A_{2}(\eta ; \zeta, \iota)}{(\iota-\zeta)^{2}}(I R) \int_{\varrho}^{\zeta}[\digamma(\xi, \zeta) \Omega(\xi, \iota)+\digamma(\xi, \iota) \Omega(\xi, \zeta)] w_{1}(\xi) d \xi \\
& +\frac{2 \mathbf{K}(\varrho, \varsigma, \zeta, \iota)}{(\varsigma-\varrho)^{2}(\iota-\zeta)^{2}} \mathcal{B}_{1}(\xi ; \varrho, \varsigma) \mathcal{B}_{2}(\eta ; \zeta, \iota) \\
& +\frac{2 \mathbf{L}(\varrho, \zeta, \zeta, \iota)}{(\varsigma-\varrho)^{2}(\iota-\zeta)^{2}} \mathcal{A}_{1}(\xi ; \varrho, \varsigma) \mathcal{B}_{2}(\eta ; \zeta, \iota) \\
& +\frac{2 \mathbf{M}(\varrho, \varsigma, \zeta, \iota)}{(\varsigma-\varrho)^{2}(\iota-\zeta)^{2}} \mathcal{B}_{1}(\xi ; \varrho, \varsigma) \mathcal{A}_{2}(\eta ; \zeta, \iota) \\
& +\frac{2 \mathbf{N}(\varrho, \zeta, \zeta, \iota)}{(\varsigma-\varrho)^{2}(\iota-\zeta)^{2}} \mathcal{A}_{1}(\xi ; \varrho, \varsigma) \mathcal{A}_{2}(\eta ; \zeta, \iota)
\end{aligned}
$$

By applying (2.3) to each integral in (3.22), we obtain the desired result.

Remark 3 If we choose $w_{1}(\xi)=1$ and $w_{2}(\eta)=1$ in Theorem 11 and Theorem 12, we get (2.6) and (2.7), respectively.

Remark 4 If we choose $w_{1}(\xi)=\frac{\alpha}{(\varsigma-\varrho)^{\alpha-1}}\left[(\varsigma-\xi)^{\alpha-1}+(\xi-\varrho)^{\alpha-1}\right]$ with $\alpha>0$ and $w_{2}(\eta)=$ $\frac{\beta}{(\iota-\zeta)^{\beta-1}}\left[(\iota-\eta)^{\beta-1}+(\eta-\zeta)^{\beta-1}\right]$ with $\beta>0$ in Theorem 11 and Theorem 12 , we get

$$
\begin{aligned}
& \frac{\Gamma(\alpha+1) \Gamma(\beta+1)}{4(\varsigma-\varrho)^{\alpha}(\iota-\zeta)^{\beta}} \\
& \quad \times\left[\mathcal{J}_{\varrho+, \zeta+}^{\alpha, \beta} \digamma(\varsigma, \iota) \Omega(\varsigma, \iota)+\mathcal{J}_{\varrho+, \ell-}^{\alpha, \beta} \digamma(\varsigma, \zeta) \Omega(\varsigma, \zeta)+\mathcal{J}_{\zeta^{-, \zeta+}+}^{\alpha, \beta} \digamma(\varrho, \iota) \Omega(\varrho, \iota)\right.
\end{aligned}
$$




$$
\begin{aligned}
& \left.+\mathcal{J}_{\zeta-,-l}^{\alpha, \beta} \digamma(\varrho, \zeta) \Omega(\varrho, \zeta)\right] \\
\supseteq & {\left[\frac{1}{2}-\frac{\beta}{(\beta+1)(\beta+2)}\right]\left[\frac{1}{2}-\frac{\alpha}{(\alpha+1)(\alpha+2)}\right] \mathbf{K}(\varrho, \zeta, \zeta, \iota) } \\
& +\left[\frac{1}{2}-\frac{\beta}{(\beta+1)(\beta+2)}\right]\left[\frac{\alpha}{(\alpha+1)(\alpha+2)}\right] \mathbf{L}(\varrho, \zeta, \zeta, \iota) \\
& +\left[\frac{\beta}{(\beta+1)(\beta+2)}\right]\left[\frac{1}{2}-\frac{\alpha}{(\alpha+1)(\alpha+2)}\right] \mathbf{M}(\varrho, \zeta, \zeta, \iota) \\
& +\left[\frac{\beta}{(\beta+1)(\beta+2)}\right]\left[\frac{\alpha}{(\alpha+1)(\alpha+2)}\right] \mathbf{N}(\varrho, \zeta, \zeta, \iota)
\end{aligned}
$$

and

$$
\begin{aligned}
4 \digamma( & \left.\frac{\varrho+\zeta}{2}, \frac{\zeta+\iota}{2}\right) \Omega\left(\frac{\varrho+\zeta}{2}, \frac{\zeta+\iota}{2}\right) \\
\supseteq & \frac{\Gamma(\alpha+1) \Gamma(\beta+1)}{4(\zeta-\varrho)^{\alpha}(\iota-\zeta)^{\beta}} \\
& \times\left[\mathcal{J}_{\varrho+, \zeta+}^{\alpha, \beta} \digamma(\zeta, \iota) \Omega(\zeta, \iota)+\mathcal{J}_{\varrho+, \iota-}^{\alpha, \beta} \digamma(\zeta, \zeta) \Omega(\zeta, \zeta)+\mathcal{J}_{\zeta-, \zeta+}^{\alpha, \beta} \digamma(\varrho, \iota) \Omega(\varrho, \iota)\right. \\
& \left.+\mathcal{J}_{\zeta-, \iota-}^{\alpha, \beta}(\varrho, \zeta) \Omega(\varrho, \zeta)\right] \\
& +\left\{\frac{\alpha}{2(\alpha+1)(\alpha+2)}+\left[\frac{\beta}{(\beta+1)(\beta+2)}\right]\left[\frac{1}{2}-\frac{\alpha}{(\alpha+1)(\alpha+2)}\right]\right\} \mathbf{K}(\varrho, \zeta, \zeta, \iota) \\
& +\left\{\frac{1}{2}\left[\frac{1}{2}-\frac{\alpha}{(\alpha+1)(\alpha+2)}\right]+\left[\frac{\alpha}{(\alpha+1)(\alpha+2)}\right]\left[\frac{\beta}{(\beta+1)(\beta+2)}\right]\right\} \mathbf{L}(\varrho, \zeta, \zeta, \iota) \\
& +\left\{\frac{1}{2}\left[\frac{1}{2}-\frac{\beta}{(\beta+1)(\beta+2)}\right]+\left[\frac{\alpha}{(\alpha+1)(\alpha+2)}\right]\left[\frac{\beta}{(\beta+1)(\beta+2)}\right]\right\} \mathbf{M}(\varrho, \zeta, \zeta, \iota) \\
& +\left\{\frac{1}{4}-\left[\frac{\alpha}{(\alpha+1)(\alpha+2)}\right]\left[\frac{\beta}{(\beta+1)(\beta+2)}\right]\right\} \mathbf{N}(\varrho, \zeta, \zeta, \iota)
\end{aligned}
$$

which are proved by Kara et al. in [4].

\section{Concluding remarks}

In this research, some new Hermite-Hadamard-Fejér type inclusions for the product of two co-ordinated convex interval-valued functions are offered. We also proved that the results given in this work generalize the results given in [4,53]. It is an interesting and new problem that the upcoming researchers can obtain similar inclusions for different kinds of convexities and integrals in their future investigation.

\section{Acknowledgements}

We are thankful to the editor and referees for their careful reading and valuable suggestions to make the article reader friendly.

\section{Funding}

The work was supported by the Natural Science Foundation of China (Grant Nos. 61673169, 11301127, 11701176, $11626101,11601485,11971241)$.

Availability of data and materials

Data sharing not applicable to this paper as no data sets were generated or analyzed during the current study. 
Authors' contributions

The study was carried out in collaboration of all authors. All authors read and approved the final manuscript.

\section{Author details}

${ }^{1}$ Department of Mathematics, Faculty of Science and Arts, Düzce University, Düzce, Turkey. ${ }^{2}$ Jiangsu Key Laboratory for NSLSCS, School of Mathematical Sciences, Nanjing Normal University, Nanjing, 210023, China. ${ }^{3}$ Department of Mathematics, Huzhou University, Huzhou 313000, China.

\section{Publisher's Note}

Springer Nature remains neutral with regard to jurisdictional claims in published maps and institutional affiliations.

Received: 24 October 2020 Accepted: 28 January 2021 Published online: 10 February 2021

\section{References}

1. Aubin, J.P., Cellina, A.: Differential Inclusions. Springer, New York (1984)

2. Breckner, W.W.: Continuity of generalized convex and generalized concave set-valued functions. Rev. Anal. Numér. Théor. Approx. 22(1), 39-51 (1993)

3. Budak, H., Bakış, Y.: On Fejér type inequalities for products two convex functions. Note Mat. (in press). https://doi.org/10.13140/RG.2.2.10003.73769

4. Budak, H., Kara, H., Ali, M.A., Khan, S.: Fractional Hermite-Hadamard type inequalities for interval-valued co-ordinated convex functions (2020, submitted)

5. Budak, H., Kara, H., Erden, S.: On Fejér type inequalities for products of interval-valued convex functions (2020, submitted)

6. Budak, H., Sarikaya, M.Z.: Hermite-Hadamard type inequalities for products of two co-ordinated convex mappings via fractional integrals. Int. J. Appl. Math. Stat. 58(4), 11-30 (2019)

7. Budak, H., Tunç, T., Sarikaya, M.Z.: Fractional Hermite-Hadamard-type inequalities for interval-valued functions. Proc Am. Math. Soc. 148(2), 705-718 (2020)

8. Chalco-Cano, Y., Flores-Franulic, A., Roman-Flores, H.: Ostrowski type inequalities for interval-valued functions using generalized Hukuhara derivative. Comput. Appl. Math. 31, 457-472 (2012)

9. Chalco-Cano, Y., Lodwick, W.A., Condori-Equice, W.: Ostrowski type inequalities and applications in numerical integration for interval-valued functions. Soft Comput. 19, 3293-3300 (2015)

10. Chen, F.: A note on Hermite-Hadamard inequalities for products of convex functions. J. Appl. Math. 2013, Article ID $935020(2013)$

11. Chen, F.: A note on Hermite-Hadamard inequalities for products of convex functions via Riemann-Liouville fractional integrals. Ital. J. Pure Appl. Math. 33, 299-306 (2014)

12. Chen, F., Wu, S.: Several complementary inequalities to inequalities of Hermite-Hadamard type for s-convex functions. J. Nonlinear Sci. Appl. 9, 705-716 (2016)

13. Costa, T.M.: Jensen's inequality type integral for fuzzy interval-valued functions. Fuzzy Sets Syst. 327, 31-47 (2017)

14. Costa, T.M., Roman-Flores, H.: Some integral inequalities for fuzzy-interval-valued functions. Inf. Sci. 420, 110-125 (2017)

15. Dinghas, A.: Zum Minkowskischen Integralbegriff abgeschlossener Mengen. Math. Z. 66, 173-188 (1956)

16. Dragomir, S.S., Agarwal, R.P.: Two inequalities for differentiable mappings and applications to special means of rea numbers and to trapezoidal formula. Appl. Math. Lett. 11(5), 91-95 (1998)

17. Erden, S., Sarıkaya, M.Z., Budak, H.: New weighted inequalities for higher order derivatives and applications. Filomat 32(12), 4419-4433 (2018)

18. Fejér, L.: Über die Fourierreihen, II. Math. Naturwiss. Anz. Ungar. Akad. Wiss. 24, 369-390 (1906) (Hungarian)

19. Flores-Franulic, A., Chalco-Cano, Y., Roman-Flores, H.: An Ostrowski type inequality for interval-valued functions. In: IFSA World Congress and NAFIPS Annual Meeting IEEE, vol. 35, pp. 1459-1462 (2013)

20. Gorenflo, R., Mainardi, F.: Fractional calculus: integral and differential equations of fractional order. In: Fractals and Fractional Calculus in Continuum Mechanics, pp. 223-276. Springer, Wien (1997)

21. Hue, N.N., Huy, D.Q.: Some inequalities of the Hermite-Hadamard type for product of two functions. J. New Theory, 26-37 (2016)

22. Iscan, I.: Hermite-Hadamard-Fejér type inequalities for convex functions via fractional integrals. Stud. Univ. Babeş-Bolyai, Math. 60(3), 355-366 (2015)

23. Kara, H., Ali, M.A., Budak, H.: Hermite-Hadamard-type inequalities for interval-valued coordinated convex functions involving generalized fractional integrals. Math. Methods Appl. Sci. (2020). https://doi.org/10.1002/mma.6712

24. Kilbas, A.A., Srivastava, H.M., Trujillo, J.J.: Theory and Applications of Fractional Differential Equations. North-Holland Mathematics Studies, vol. 204. Elsevier, Amsterdam (2006)

25. Kırmacl, U.S.: Inequalities for differentiable mappings and applications to special means of real numbers and to midpoint formula. Appl. Math. Comput. 147, 137-146 (2004)

26. Kırmacı, U.S., Bakula, M.K., Özdemir, M.E., Pečarić, J.: Hadamard-type inequalities for s-convex functions. Appl. Math. Comput. 193, 26-35 (2007)

27. Latif, M.A., Alomari, M.: Hadamard-type inequalities for product two convex functions on the co-ordinates. Int. Math Forum 4(47), 2327-2338 (2009)

28. Liu, X., Ye, G., Zhao, D., Liu, W.: Fractional Hermite-Hadamard type inequalities for interval-valued functions. J. Inequal. Appl. 2019(1), 266 (2019)

29. Lupulescu, V.: Fractional calculus for interval-valued functions. Fuzzy Sets Syst. 265, 63-85 (2015)

30. Markov, S.: On the algebraic properties of convex bodies and some applications. J. Convex Anal. 7(1), 129-166 (2000)

31. Miller, S., Ross, B.: An Introduction to the Fractional Calculus and Fractional Differential Equations p. 2. Wiley, New York (1993) 
32. Mitroi, F.C., Kazimierz, N., Wasowicz, S.: Hermite-Hadamard inequalities for convex set-valued functions. Demonstr. Math. XLVI(4), 655-662 (2013)

33. Moore, R.E.: Interval Analysis. Prentice Hall, Englewood Cliffs (1966)

34. Moore, R.E., Kearfott, R.B., Cloud, M.J.: Introduction to Interval Analysis, vol. 110. SIAM, Philadelphia (2009)

35. Nikodem, K.: On midpoint convex set-valued functions. Aequ. Math. 33, 46-56 (1987)

36. Nikodem, K., Snchez, J.L., Snchez, L.: Jensen and Hermite-Hadamard inequalities for strongly convex set-valued maps. Math. Æterna 4(8), 979-987 (2014)

37. Osuna-Gómez, R., Jiménez-Gamero, M.D., Chalco-Cano, Y., Rojas-Medar, M.A.: Hadamard and Jensen inequalities for s-convex fuzzy processes. In: Soft Methodology and Random Information Systems, pp. 645-652. Springer, Berlin (2004)

38. Ozdemir, M.E., Latif, M.A., Akdemir, A.O.: On some Hadamard-type inequalities for product of two s-convex functions on the co-ordinates. J. Inequal. Appl. 2012, 21 (2012)

39. Ozdemir, M.E., Latif, M.A., Akdemir, A.O.: On some Hadamard-type inequalities for product of two $h$-convex functions on the co-ordinates. Turk. J. Sci. 1, 41-58 (2016)

40. Pachpatte, B.G.: On some inequalities for convex functions. RGMIA Res. Rep. Coll. 6(E) (2003)

41. Piatek, B.: On the Sincov functional equation. Demonstr. Math. 38(4), 875-882 (2005)

42. Piatek, B.: On the Riemann integral of set-valued functions. Zeszyty Naukowe. Matematyka Stosowana/Politechnika Slaska (2012)

43. Podlubni, I.: Fractional Differential Equations. Academic Press, San Diego (1999)

44. Roman-Flores, H., Chalco-Cano, Y., Lodwick, W.A.: Some integral inequalities for interval-valued functions. Comput. Appl. Math. 37, 1306-1318 (2018)

45. Roman-Flores, H., Chalco-Cano, Y., Silva, G.N.: A note on Gronwall type inequality for interval-valued functions. In: IFSA World Congress and NAFIPS Annual Meeting IEEE, vol. 35, pp. 1455-1458 (2013)

46. Sadowska, E.: Hadamard inequality and a refinement of Jensen inequality for set-valued functions. Results Math. 32 , 332-337 (1997)

47. Sarikaya, M.Z., Erden, S.: On the Hermite-Hadamard-Fejér type integral inequality for convex function. Turk. J. Anal. Number Theory 2(3), 85-89 (2014)

48. Sarikaya, M.Z., Erden, S.: On the weighted integral inequalities for convex functions. Acta Univ. Sapientiae Math. 6(2), 194-208 (2014)

49. Sarikaya, M.Z., Set, E., Yaldiz, H., Basak, N.: Hermite-Hadamard's inequalities for fractional integrals and related fractional inequalities. Math. Comput. Model. 57, 2403-2407 (2013)

50. Set, E., Özdemir, M.E., Dragomir, S.S.: On the Hermite-Hadamard inequality and other integral inequalities involving two functions. J. Inequal. Appl. 2010, Article ID 148102 (2010)

51. Wu, Y., Qi, F., Niu, D.-W.: Integral inequalities of Hermite-Hadamard type for the product of strongly logarithmically convex and other convex functions. Maejo Int. J. Sci. Technol. 9(3), 394-402 (2015)

52. Yin, H.-P., Qi, F.: Hermite-Hadamard type inequalities for the product of $(\alpha, m)$-convex functions. J. Nonlinear Sci. Appl. 8, 231-236 (2015)

53. Zhao, D., Ali, M.A., Murtaza, G., Zhang, Z:: On the Hermite-Hadamard inequalities for interval-valued co-ordinated convex functions. Adv. Differ. Equ. 2020, 570 (2020)

54. Zhao, D., An, T., Ye, G., Liu, W.: New Jensen and Hermite-Hadamard type inequalities for $h$-convex interval-valued functions. J. Inequal. Appl. 2018, 302 (2018)

55. Zhao, D., An, T., Ye, G., Liu, W.: Chebyshev type inequalities for interval-valued functions. Fuzzy Sets Syst. 396, 82-101 (2020)

56. Zhao, D., Ye, G., Liu, W., Torres, D.F.: Some inequalities for interval-valued functions on time scales. Soft Comput. 23, 6005-6015 (2019)

\section{Submit your manuscript to a SpringerOpen ${ }^{\circ}$ journal and benefit from:}

- Convenient online submission

- Rigorous peer review

- Open access: articles freely available online

- High visibility within the field

- Retaining the copyright to your article

Submit your next manuscript at $\gg$ springeropen.com 